\title{
The growth of resonant tunneling hot electron transistors using chemical beam epitaxy
}

\author{
W.L. Chen, G.O. Munns, L. Davis, P.K. Bhattacharya and G.I. Haddad \\ Center for High Frequency Microelectronics, Solid-State Electronics Laboratory, Department of Electrical Engineering and Computer \\ Science, The Unicersity of Michigan, Ann Arbor, Michigan 48109-2122, USA
}

\begin{abstract}
A systematic growth study of InGaAs/AlAs/InGaAsP resonant tunneling hot electron transistors (RHETs) was performed using chemical beam epitaxy (CBE). The resonant tunneling hot electron transistors studied consist of a highly strained AlAs $/ \ln _{0.75} \mathrm{Ga}_{0.25} \mathrm{As} / \mathrm{AlAs}$ double barrier structure and an undoped InP collector barrier with 1.1 and $1.2 \mu \mathrm{m}$ InGaAsP graded layers. These quaternaries were lattice matched to $\mathrm{InP}$ within $2.6 \times 10^{-4}$ and showed an averaged full width at half-maximum (FWHM) of $6 \mathrm{meV}$ from low temperature photoluminescence (PL) measurement. The effects of growth interrupt were studied using PL, X-ray diffraction and secondary ion mass spectrometry (SIMS) measurements. It was found that excessive growth interrupt induced high oxygen accumulation $\left(8 \times 10^{18} \mathrm{~cm}^{-3}\right)$ at the heterojunction and reduced the intensity of PL spectra. Moreover, for the growth of tunneling heterostructures, low substrate temperature, appropriate growth interrupts and use of hydride drying filters and high purity hydrides were helpful to improve device performance. The highest peak-to-valley current ratio (PVR) observed was 12.7 , and maximum base transport ratio was 0.98 at $80 \mathrm{~K}$. Furthermore, some digital functions such as flip-flop gate and exclusive NOR were demonstrated using a single RHET.
\end{abstract}

\section{Introduction}

Chemical beam epitaxy (CBE) combines the versatility of the vapor sources of metalorganic chemical vapor deposition (MOCVD) with the molecular beam properties of MBE. Based on such unique features [1], CBE has been applied to grow a wide range of devices, including (1) optoelectronic devices such as InGaAsP lasers [2], Bragg reflectors [3], (2) electronic devices such as C-doped heterojunction bipolar transistors (HBTs) [4] and InP channel high electron mobility transistors (HEMTs) [5]. All these applications show promising high speed device performance, because CBE is capable of growing Pcontaining compounds and InP lattice matched materials with abrupt heterojunctions, offering superior transport properties. In order to provide a higher level device speed, resonant tunneling devices are needed for next generation devices and circuits. However, to our knowledge, there is no investigation of such devices using CBE. In this work, the main effort is to grow high quality bulk materials and heterojunctions for resonant tunneling devices.

A systematic growth study was performed to realize resonant tunneling hot electron transistors (RHETs), including (1) the characterization of bulk material, including $\operatorname{In}_{0.75} \mathrm{Ga}_{0.25}$ As and 1.1 and $1.2 \mu \mathrm{m}$ InGaAsP; (2) the investigation of the effects of growth interrupt; (3) the influence of using hydride drying filters and high purity hydrides; (4) the optimization of growth conditions for resonant tunneling diodes (RTDs) and RHETs; (5) the measurement of device and circuit performance.

\section{Experiments}

The structures studied were grown using an Intevac Gen II CBE reactor. The structures were grown on (100) $\mathrm{Fe}$-doped semi-insulating $\mathrm{InP}$ substrates at a substrate temperature of $490^{\circ} \mathrm{C}$. The source materials were trimethylindium (TMI), triethylgallium (TEG), trimethylamine 
alane (TMAA), 100\% phosphine $\left(\mathrm{PH}_{3}\right)$ and $100 \%$ arsine $\left(\mathrm{AsH}_{3}\right)$ for the InGaAs, InP, InGaAsP and AlAs. Si was used for the n-type dopant.

First, bulk material was characterized with double crystal X-ray diffraction measurement for desired alloy composition. For example, (400) and (511) X-ray diffraction [6] was used to calibrate the $\mathrm{In}_{0.75} \mathrm{Ga}_{0.25}$ As compound. Second, 1.1 and 1.2 $\mu \mathrm{m}$ InGaAsP were calibrated. Third, several InGaAs/InAlAs multiple quantum well structures were grown with growth interrupts at either or both junctions. To estimate the effects of growth interrupt, X-ray, SIMS and low temperature PL measurements were performed. Fourth, the optimal growth interrupt condition was applied to grow a highly strained $\mathrm{AlAs} / \mathrm{In}_{0.75} \mathrm{Ga}_{0.25} \mathrm{As} /$ AlAs double barrier structure with different substrate temperatures for comparison of interface roughness. Finally, several RHETs were grown using the information obtained from the growth study. Moreover, during the growth of RHET and RTD structures, hydride drying filters were turned on and off to check their influences.

The fabrication of the RTDs was carried out using conventional photo-lithography, wet chemical mesa etching and metal lift-off process. That of the RHETs was performed using a novel selfaligned transistor process. Then a single RHET and external resistors were connected to build the digital circuits studied.

\section{Results and discussion}

\subsection{Characterization of bulk materials}

First, all bulk materials needed were calibrated for acceptable surface morphology and mobility. The growth rate of bulk materials, including InP, AlAs, $\mathbf{I n}_{0.53} \mathrm{Ga}_{0.47} \mathrm{As}, \mathrm{In}_{0.75} \mathrm{Ga}_{0.25} \mathrm{As}$ and InGaAsP, were measured using selective chemical etching. Then the thicknesses of the barrier and quantum well were initially estimated according to the information obtained from the bulk materials. Then such estimation was confirmed with PL and device results.

In order to reduce the quantum reflection of electrons at the base/collector junction of a RHET, 1.1 and $1.2 \mu \mathrm{m}$ InGaAsP were calibrated to grade the collector barrier. The optimal growth conditions used were as follows: the group III total flow was held constant at 1.25 SCCM, the $\mathrm{V} / \mathrm{III}$ ratio was 20 and the optimal growth temperature was $480^{\circ} \mathrm{C}$. The bulk materials calibrated showed good qualities from X-ray and low temperature PL measurements. The double crystal X-ray rocking curve of a typical $1.1 \mu \mathrm{m}$ InGaAsP is shown in fig. 1a, indicating clear pendellösung fringes and lattice matching to InP within 70 arc $\sec \left(|\Delta a / a|<2.6 \times 10^{-4}\right)$. The PL measurement was performed at $18 \mathrm{~K}$, as shown in fig. $1 \mathrm{~b}$, showing a FWHM of $5.6 \mathrm{meV}$.
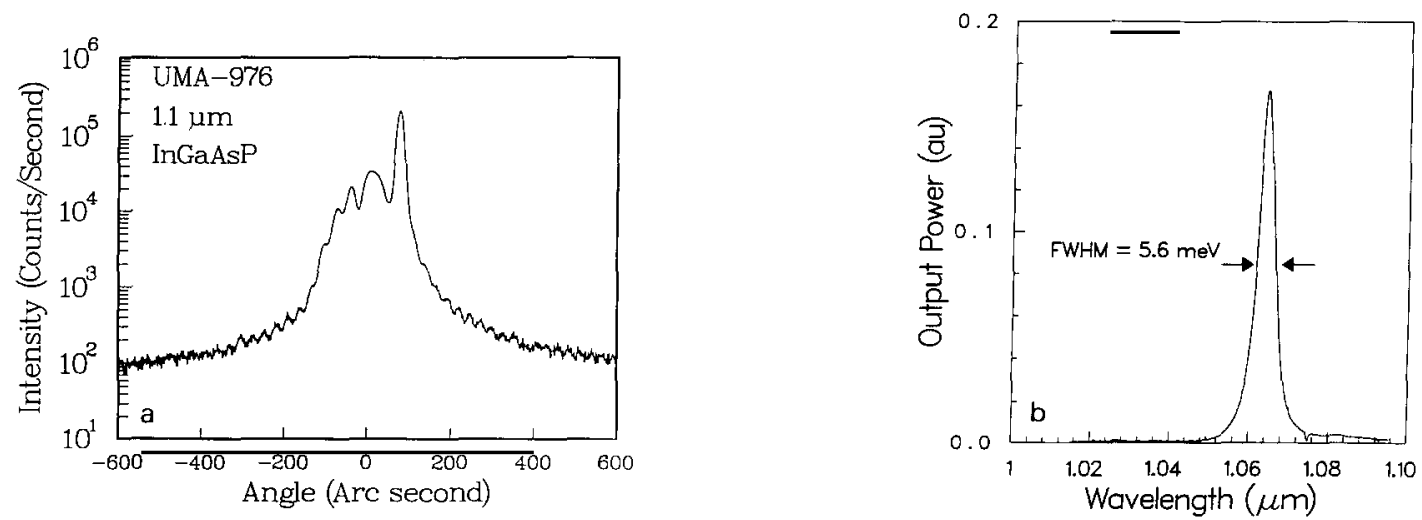

Fig. 1. (a) X-ray rocking curve of the grown $1.1 \mu \mathrm{m}$ InGaAsP lattice matched to InP. (b) PL spectra at $18 \mathrm{~K}$ of the grown $1.1 \mu \mathrm{m}$ InGaAsP lattice matched to InP. 
A previous study [7] had determined the optimal growth conditions for bulk InP, InGaAs and InP/InGaAs, InGaAs/InP heterojunctions. Using hydride drying filters in conjunction with high purity hydrides was helpful to improve material properties [8]. The highest InP mobility with excellent surface morphology is $90,000 \mathrm{~cm}^{2} / \mathrm{V} \cdot \mathrm{s}$ at $77 \mathrm{~K}$ for a substrate temperature of $520^{\circ} \mathrm{C}$. It is found that the InP surface morphology becomes worse as the substrate temperature increases, however electron mobility increases as well. The InGaAs surface morphology and mobility showed little dependence on growth conditions over the range investigated, but lattice match is sensitive to growth temperature. In order to get the optimal growth conditions for different compounds, growth interrupt was necessary to ramp the substrate temperature.

\subsection{Growth interrupt}

During the growth of RTD structures, the technique used to switch between well and barrier materials can have significant effects on the geometrical shapes of RTDs, and the sharpness of the interfaces between well and barrier layers. These effects become more dramatic for CBE when gases are used as source materials. The switching sequence study optimized the group III change during the growth of a highly strained AlAs $/ \operatorname{In}_{0.75} \mathrm{Ga}_{0.25}$ As / AlAs double barrier structure. Therefore, several InGaAs/InAlAs multiquantum well structures lattice matched to InP, as shown in fig. $2 \mathrm{a}$, were grown to estimate the effects of growth interrupt. Two different lengths of interrupt were used, $5 \mathrm{~s}$ and $125 \mathrm{~s}$, at either or both junctions.

The effects of growth interrupt were first estimated using SIMS measurement. As shown in fig. $2 \mathrm{~b}, 125 \mathrm{~s}$ interrupt at both junctions induces high oxygen accumulation $\left(\sim 8 \times 10^{18} \mathrm{~cm}^{-3}\right)$ at the InGaAs(top)/InAlAs junction, indicating oxidation of InAlAs. Changing the interrupt length to $5 \mathrm{~s}$ reduces the oxygen accumulation by about $40 \%$. Low temperature (18 K) PL measurement shows that such oxygen impurities act as recombination centers, reducing the intensity of PL spectra by $55 \%$ and broadening the linewidth of PL
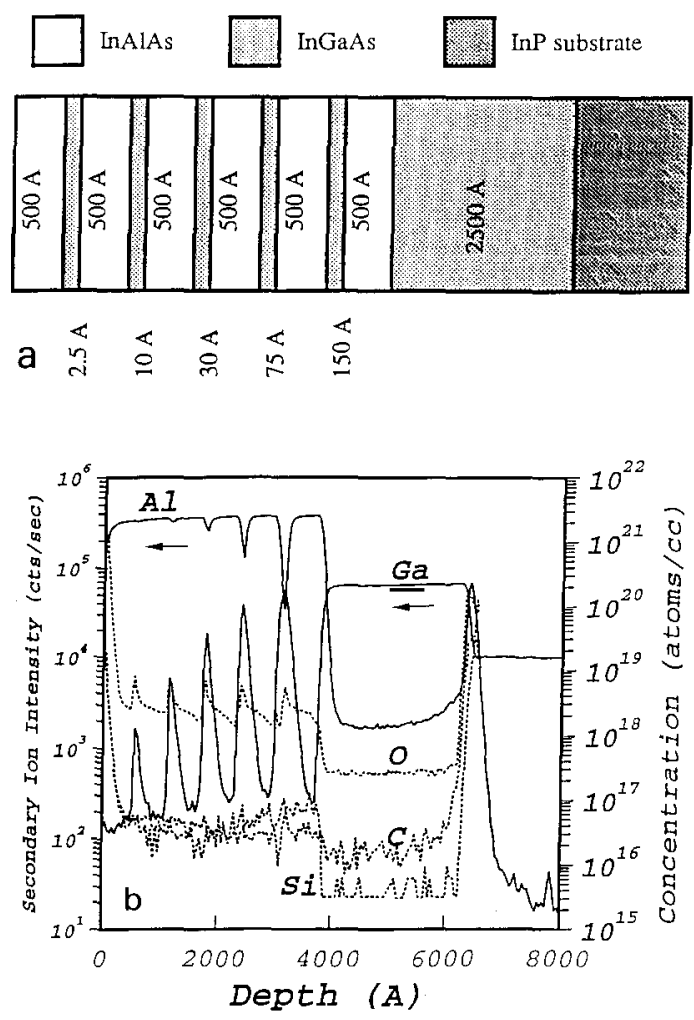

Fig. 2. (a) $\mathrm{In}_{0.52} A \mathrm{I}_{0.48} \mathrm{As} / \mathrm{In}_{0.53} \mathrm{Ga}_{0.47} \mathrm{As}$ MQW structure for the growth interrupt study. (b) SIMS results for the MQW structure with $125 \mathrm{~s}$ growth interrupt at each heterojunction. High oxygen accumulation is found at the InGaAs (top)/In AlAs junction.

spectra. Finally, all the samples grown were tested using double crystal X-ray measurement and all of them showed clear pendellösung fringes. There is no significant difference in X-ray spectra for samples grown with different lengths of interrupt.

The information obtained was subsequently applied to the growth of the highly strained AlAs $/ \operatorname{In}_{0.75} \mathrm{Ga}_{0.25}$ As/AIAs $(20 / 45 / 20 \AA)$ double barrier structure. With $5 \mathrm{~s}$ growth interrupt at each heterojunction, the RTD grown did not show either clear pendellösung fringes from X-ray measurement or negative differential resistance (NDR) from the $I-V$ characteristics. It is believed that the RTDs with such thin AlAs barriers are sensitive to the roughness of interfaces. In order to reduce the incorporation of impurities and smooth the growth front, an additional $120 \mathrm{~s}$ 
growth interrupt was introduced at the point about 10 monolayers before the growth of the AlAs barrier. Normally, the RTDs grown with such interrupt sequences showed both NDRs and pendellösung fringes. In general, the X-ray diffraction patterns show clear pendellösung fringes for those RTDs or RHETs which work well electrically.

\subsection{Hydride filters, high purity hydrides and sub- strate temperature}

In this section, the influences of hydride drying filters, high purity hydrides [8] and substrate temperatures on the RTD and RHET performance were investigated. All the RTDs were grown with the growth interrupts described in the previous section. First, hydride drying filters were installed in the arsine and phosphine lines to remove water and oxygen to less than 10 parts per billion (PPB). For those RTDs grown at $500^{\circ} \mathrm{C}$, using drying filters and high purity hydrides improved the peak-to-valley current ratios (PVRs) from 6 to 10 at $300 \mathrm{~K}$. For RHETs without the InGaAsP graded layer, the PVRs increased from 10 to 20 and the current gain also improved from 11 to 24 at $80 \mathrm{~K}$. The improvement of the device performance is attributed to the reduction of the oxygen background source, resulting in less impurity incorporation in the AlAs barriers. Thereby, the scattering centers in the AlAs barriers are reduced significantly and resonant electrons can preserve better coherence inside the double barrier region. The leakage current or valley current of the RTDs drops significantly, increasing the PVRs of the RTDs and RHETs. The increase of RHET current gain is due to the reduced scattering centers in the base and the highly coherent tunneling electrons from the emitter.

The effects of the substrate temperature on the RTD $I-V$ characteristics were studied at three different substrate temperatures, 540, 520 and $500^{\circ} \mathrm{C}$. For the same RTD structure, high substrate temperature $\left(540^{\circ} \mathrm{C}\right)$ results in resistive $I-V$ characteristics and no NDRs observed, even though the growth interrupt sequences were used. Lowering the substrate temperature to 520 and $500^{\circ} \mathrm{C}$, NDRs were observed in the $I-V$ charac-

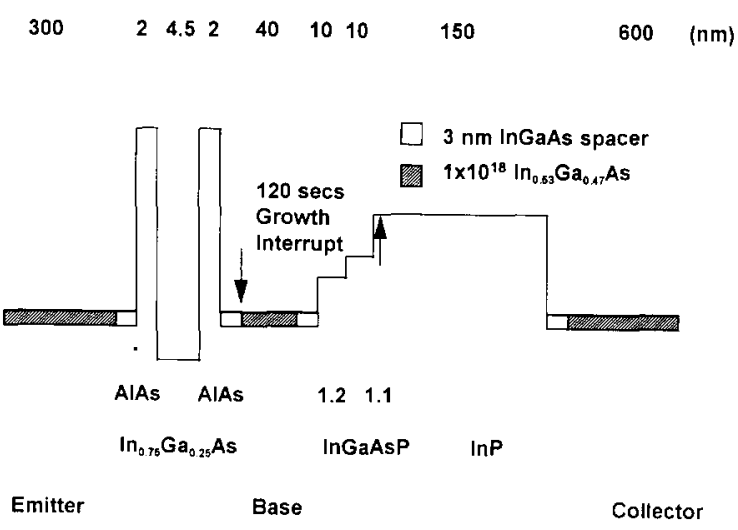

Fig. 3. The energy band diagram for the InGaAs/AlAs/In GaAsP RHET studied. Growth sequence is also shown.

teristics measured. The possible reason for such temperature dependent effects is the indium surface segregation problem, which is enhanced by the high temperature growth $[9,10]$. During the growth from $\mathrm{In}_{0.75} \mathrm{Ga}_{0.25}$ As to $\mathrm{AlAs}$, a $540^{\circ} \mathrm{C}$ substrate temperature induces serious indium segregation and makes this heterointerface relatively rough, resulting in the destruction of the coherence of resonant tunneling electrons. It is believed that such high growth temperature also causes the same problems during the growth from InGaAs to AlAs. Therefore, no smooth heterojunction can be detected from the double crystal $\mathrm{X}$-ray measurement, resulting in the X-ray rocking curves without clear pendellösung fringes. Lowering the substrate to 520 and $500^{\circ} \mathrm{C}$ reduced this indium surface segregation problem.

\subsection{RHETS}

After the growth study, RHETs with a In GaAsP graded collector barrier were grown in a sequence as shown in fig. 3. As mentioned, there is a $5 \mathrm{~s}$ growth interrupt at each heterojunction. Before the growth of 1.1 and $1.2 \mu \mathrm{m}$ InGaAsP, a growth interrupt of $120 \mathrm{~s}$ was used to ramp the substrate temperature from 520 to $480^{\circ} \mathrm{C}$. The substrate temperature was changed to $500^{\circ} \mathrm{C}$ before the growth of the highly strained AlAs/ $\mathrm{In}_{0.75} \mathrm{Ga}_{0.25} \mathrm{As} / \mathrm{AlAs}$ system, thus a $120 \mathrm{~s}$ growth interrupt was used again. 


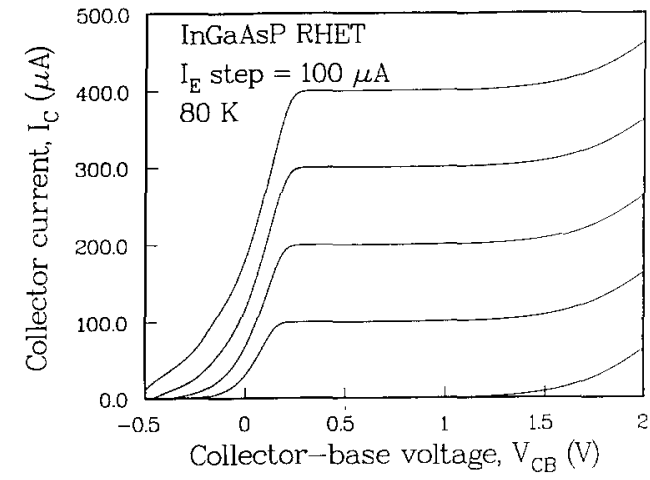

Fig. 4. The RHET common base $I-V$ characteristics at $80 \mathrm{~K}$. $\alpha=0.98$.

The common base $I-V$ characteristics of the RHET studied are shown in fig. 4 , indicating a common base transfer ratio of 0.98 . The transfer $I-V$ characteristics shows the highest PVR of around 12.7 with a peak current density of $2 \times 10^{4}$ $\mathrm{A} / \mathrm{cm}^{2}$ at $80 \mathrm{~K}$, as shown in fig. 5 . Based on the negative differential transconductance characteristics, the RHET can be connected with several external resistors to form an exclusive-NOR function, as shown in fig. 6. Several MESFETs or other types of transistors are conventionally required to build such a logic function gate. A flip-flop gate was also demonstrated using a single RHET. Moreover, the highest cutoff fre-

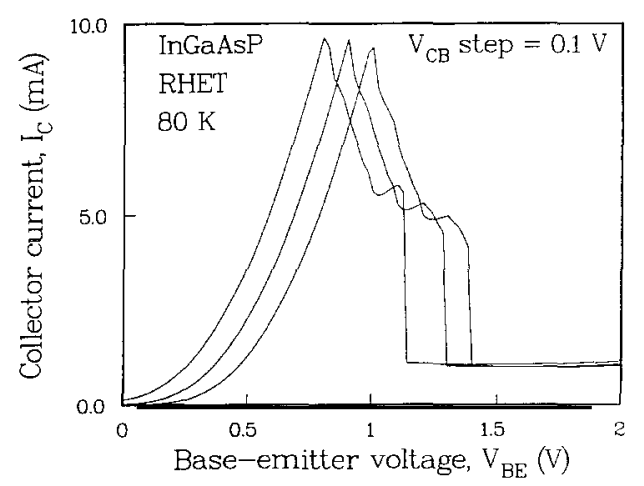

Fig. 5. The RHET transfer $I-V$ characteristics at $80 \mathrm{~K}$. PVR $=12.7$.
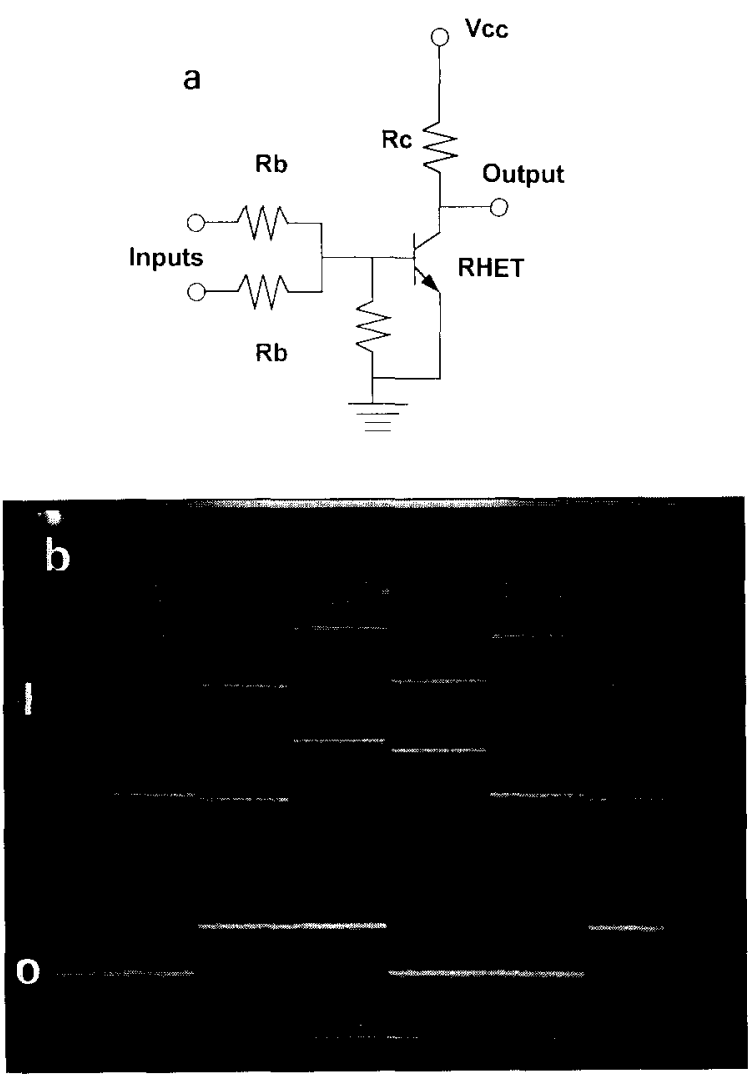

Fig. 6. (a) The RHET exclusive-NOR circuit and (b) observed traces at $80 \mathrm{~K}$ (I: inputs, $1 \mathrm{~V} / \mathrm{div}$; O: output, $0.5 \mathrm{~V} / \mathrm{div}$ ).

quency $\left(f_{\mathrm{T}}\right)$ measured is $31 \mathrm{GHz}$ at room temperature.

\section{Conclusion}

In this study, a systematic growth study was performed to realize InP-based RHETs with In GaAsP graded collector barriers using CBE for the first time. The $1.1 \mu \mathrm{m}$ and $1.2 \mu \mathrm{m}$ InGaAsP grown showed excellent material quality, with an average FWHM of $6 \mathrm{meV}$ from PL measurements at $18 \mathrm{~K}$. Appropriate growth interrupt was found to be necessary to realize RTDs and RHETs. Excess interrupt induced high oxygen accumulation in the $\mathrm{Al}$ containing compounds, reducing the intensity of the PL spectra. Using hydride drying filters and high purity hydrides 
improve the PVRs of the RTDs and RHETs about 2 times. Finally, the RHET studied showed the highest PVR of 12.7 and a base transfer ratio of 0.98 at $80 \mathrm{~K}$. An exclusive-NOR function was also demonstrated using a single RHET.

\section{Acknowledgment}

This work was supported by the US Army Research Office under the URI program contract DAAL03-92-G-0109.

\section{References}

[1] W.T. Tsang, J. Crystal Growth 120 (1992) 1.

[2] W.T. Tsang, F.S. Choa, M.C. Wu, Y.K. Chen, A.M.
Sergent and P.F. Sciortino, Jr., Appl.Phys. Letters 58 (1991) 2610.

[3] F.S. Choa, K. Tai, W.T. Tsang and S.N.G. Chin, Appl Phys. Letters 59 (1991) 1540

[4] C.R. Abernathy, F. Ren, S.J. Pearton, T.R. Fullowan, R.K. Montgomery, P.W. Wisk, J.R. Lothian and P.R. Smith, J. Crystal Growth 120 (1992) 234

[5] G.O. Munns, M.E. Sherwin, T. Brock, G.I. Haddad, Y. Kwon, G.I. Ng and D. Pavlidis, J. Crystal Growth 120 (1992) 184.

[6] Y.C. Chen, private discussion.

[7] M. Sherwin, G.O. Munns, M.E. Elta, E.G. Woelk, S.B. Cray, F.L. Terry and G. I. Haddad, J. Crystal Growth 111 (1992) 594.

[8] G.O. Munns, W.L. Chen, M.E. Sherwin, D. Knightly, G.I. Haddad, L. Davis and P.K. Bhattacharya, J. Crystal Growth 136 (1994) 166.

[9] E.C. Larkins, W. Rothemund, M. Maier, Z.M. Wang, J.D. Ralston and W. Jantz, J. Crystal Growth 127 (1993) 541.

[10] K. Muraki, S. Fukatsu, Y. Shiraki and R. Ito, J. Crystal Growth 127 (1993) 546. 